MATHEMATICAL ASSOCIATION

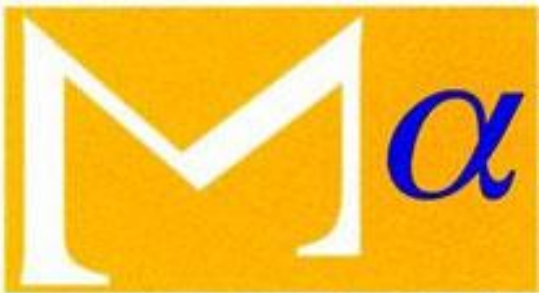

supporting mathematics in education

Notes on the Radix Method of Calculating Logarithms

Author(s): Sydney Lupton

Source: The Mathematical Gazette, Vol. 7, No. 106 (Jul., 1913), pp. 147-150

Published by: Mathematical Association

Stable URL: http://www.jstor.org/stable/3605088

Accessed: 06-01-2016 06:21 UTC

Your use of the JSTOR archive indicates your acceptance of the Terms \& Conditions of Use, available at http://www.jstor.org/page/ info/about/policies/terms.jsp

JSTOR is a not-for-profit service that helps scholars, researchers, and students discover, use, and build upon a wide range of content in a trusted digital archive. We use information technology and tools to increase productivity and facilitate new forms of scholarship. For more information about JSTOR, please contact support@jstor.org. 


\section{NOTES ON THE RADIX METHOD OF CALCULATING LOGARI'THMS.}

IT occasionally happens that logarithms and anti-logarithms are required to many figures, and in default of tables must be calculated. If a series of such logarithms are required, they are probably most easily obtained by interpolation between numbers, the logarithms of which have been calculated to many figures by Briggs, Gardiner, Sharp and others.

If only a few values are required, this method is practically inapplicable, since it is tedious and requires somewhat large tables. Many ingenious methods have been suggested to meet such cases, but in general they necessitate special artifices and tables, which require considerable practice to use with safety.

The simplest and most generally useful method has long been known; but it has been frequently rediscovered, often with inconvenient modifications, and claimed by mathematicians who were not acquainted with the labours of their predecessors.

The method requires the aid of a table of radices or numbers of the form $\left(1+\frac{r}{10^{n}}\right)$ with their logarithms to one or two places beyond those actually required.

Such a table was given by Henry Briggs in the Arithmetica Logarithmica, cap. xiv. 1624, in which $r$ and $n$ are given from 1 to 9 , and the logarithms are calculated to fifteen places. Hence he is without doubt the true discoverer of the method, and, with two exceptions, subsequent modifications have always been for the worse.

To obtain the number corresponding to a given logarithm, the logarithms of the radices are subtracted successively until the remainder consists of ciphers. The product of the radices gives the required number. This process is still in general use.

Briggs points out that, when half the digits are reduced to ciphers, the last table can be used for the remaining digits. Hence the tables of radices need only extend to half the digits in the number.

To find the logarithm corresponding to a given number, the radices are found by repeated division. The sum of the logarithms of the first figures and of the radices gives the required logarithm.

The following examples are abbreviated from the Arithmetica Logarithmica:

To find $\log 3041 \cdot 851528656$ divide by 3041 , etc.

$$
\begin{aligned}
& 3041 \text { ) } 3041851528656 \text { ( } 1.0002 \\
& 30416082 \\
& 30416082) \overline{243328656} \text { ( } 00008 \\
& 243328656 \\
& \log 3041=3.4830164201 \\
& \log 1 \cdot 0002=868502 \\
& \log 1 \cdot 00008=\quad 347421
\end{aligned}
$$

Again, to find the logarithm of 234547721933616 .

$$
\begin{array}{r}
234) 234547721933616(1.002 \\
\frac{234468}{234} \\
468) 797219(\cdot 0003 \\
703404 \\
2345383404) \begin{array}{l}
9381533616(\cdot 00004 \\
9381533616
\end{array}
\end{array}
$$

The required $\log$ is $\log 234+\log (1 \cdot 002)+\log (1 \cdot 0003)+\log (1 \cdot 00004)$. 
When the digits in the divisor are identical with half the digits in the number the process can be continued by ordinary division.

Briggs does not give any explanation of his method of obtaining the radices, but the scheme is given and fully discussed by Gray.

If $N$ be any number the logarithm of which is required,

$$
N=10^{ \pm c} \times R^{ \pm 1} \times\left(1+N_{1}\right) \text {. }
$$

In the case of natural logarithms $10^{ \pm c}$ is found from a table of the multiples of $2 \cdot 302585 \ldots$; in the case of common logarithms $c$ is the whole or part of the characteristic, and can be supplied at sight. $R$ is a whole number which can be used either as a divisor or multiplier, it must in practice be only taken to such a number of digits that its logarithm is known or can be easily calculated. Briggs assumed the use of his general table to fourteen places, hence $R$ could be used to four figures. It is now generally taken to one, two, or three digits.

$R\left(1+N_{1}\right)$ can be further decomposed as follows :

$$
\begin{aligned}
&R) R\left(1+N_{1}\right)\left(1+r_{1}\right. \\
&\left.R\left(1+r_{1}\right)\right) \frac{R\left(1+r_{1}\right)}{N_{2}\left(r_{2}\right.} \\
&\left.R\left(1+r_{1}\right)\left(1+r_{2}\right)\right) \frac{R\left(1+r_{1}\right) r_{2}}{N_{3}} \\
& \frac{R\left(1+r_{1}\right)\left(1+r_{2}\right) r_{3}}{N_{4}}
\end{aligned}\left(r_{3}\right.
$$

It will be noticed that $N_{3}$ is the remainder after subtracting

$$
R\left(1+r_{1}\right)+R\left(1+r_{1}\right) r_{2} \text { or } R\left(1+r_{1}\right)\left(1+r_{2}\right)
$$

from $R\left(1+N_{1}\right)$ and so on ; hence finally, when $N_{n}$ is preceded by ciphers equal in number to the digits required,

$$
\begin{aligned}
N & =R\left(1+r_{1}\right)\left(1+r_{2}\right)\left(1+r_{3}\right)(\ldots), \\
\log N & =\log R+\log \left(1+r_{1}\right)+\log \left(1+r_{2}\right)+\log \left(1+r_{3}\right)+
\end{aligned}
$$

to the requisite degree of accuracy.

Though Briggs does not give any special method for calculating the logarithms of radices, he incidentally gives several examples in finding the logarithms of primes. Thus 2 is raised to the tenth power 1024, the logarithm of which is calculated as follows: The successive square roots of 2 are found until the forty-seventh gives 1.00000000000000016851 605705394977 , the decimal part of which multiplied by $2^{47}$ and the modulus gives $0.010299956639811952,6527744$ for $\log 1024$ true to eighteen places.

This method is excessively laborious, since each extraction only divides the decimal part by rather over two, and the extractions for each prime number must be continued until the number of ciphers following the decimal place is equal to the number of digits required in the logarithm, though two or three more places may be trusted. The number of figures in each root must be at least twice the number of figures required in the logarithm, since any error is much enhanced by the final multiplication.

To avoid the successive extraction of square roots in each separate case, a table of the successive square roots of ten down to the hundred and twentieth (sixty of which were printed) was prepared by Callet, 1783, which is used as follows:

Place the decimal point after the first figure of the given number. Divide the number and each quotient by the next lower root of ten, until half the required number of digits are ciphers, subtract 1 and multiply the remainder by the modulus, add the logarithms of the divisors. 
Tedious as even this method is, it seems to have been used quite recently by computers, since it is reprinted in an edition of Callet, 1906 !

Napier, Briggs and their immediate followers considered logarithms as a series of ratios or continued proportions, but it was shown by Gregory St. Venant in the Opus Geometricum, 1647, that, if the equation of the hyperbola be referred to its asymptotes as axes and the abscissae be taken in continued geometrical progression, the hyperbolic trapezia standing on the abscissae are equal. So that any complete trapezium is the logarithm of its terminal abscissa.

The quadrature of the hyperbola was first obtained but not published by Newton in 1665 or 6 . It was given by James Gregory in the Vera Circuli et Hyperbolae Quadratura, 1667, and again in his Exercitationes Geometriae, 1668. Nicholas Mercator also showed in his Logarithmotechnica, 1668, that in a right-angled Hyperbola the area of a trapezium standing on any abscissa is expressed by $x-\frac{x^{2}}{2}+\frac{x^{3}}{3}-\frac{x^{4}}{4}$, etc., which therefore expresses $\log (1+x)$.

In 1695 Halley defined logarithms as "numeri rationem exponentes," and it is from this point of view, as exponents of a base generally either $e=2 \cdot 71828 \ldots$ or 10 , that logarithms are now generally investigated.

Perhaps the three most generally useful series are

$$
\log (1 \pm x)= \pm x-\frac{x^{2}}{2} \pm \frac{x^{3}}{3}-\frac{x^{4}}{4} \pm \text { etc., }
$$

which shows that when $x$ is so small that $x^{2}$ may be neglected, $\log (1 \pm x)=x$. If $x$ be any digits following $k$ ciphers, the value of $x$ must be less than $\frac{1}{10^{k}}$, and the second term of the series must be less than $0.0^{2 k} 5$ for natural and $0.0^{2 k} 217$ for common logarithns. Hence Briggs' rule follows.

By subtraction $\log \frac{1+x}{1-x}=2\left(x+\frac{x^{3}}{3}+\frac{x^{5}}{5}+\right.$ etc. $)$, and writing $\frac{x}{a}$ for $x$,

$$
\log (a+x)-\log (a-x)=\frac{2 x}{a}+\frac{1}{12}\left(\frac{2 x}{a}\right)^{3}+\frac{1}{80}\left(\frac{2 x}{a}\right)^{5}+\text { etc. }
$$

Writing $\frac{ \pm x}{a \pm x}$ for $x, \frac{1+x}{1-x}$ becomes $\frac{a \pm x}{a}$,

$$
\log (a \pm x)-\log a= \pm 2\left(\frac{x}{2 a \pm x}+\frac{1}{3}\left(\frac{x}{2 a \pm x}\right)^{3}+\frac{1}{5}\left(\frac{x}{2 a \pm x}\right)^{5}+\text { etc. }\right)
$$

The logarithms given by these series are natural or referred to the base $e$, to convert them into common logarithms referred to the base 10 ; the nonlogarithmic expressions must be divided by $\log _{e} 10$ or $2330259 \ldots$ or multiplied by the modulus $\mu=0.42329 \ldots$ or $43 / 99$ nearly.

Thus, to find the logarithm of the radix $\left(1+\frac{9}{10^{12}}\right)$,

$$
\begin{aligned}
& \log \left(1+\frac{9}{10^{12}}\right)=\frac{9 \mu}{10^{12}}-\frac{81 \mu}{2 \cdot 10^{24}}+\text { by (i). } \\
& 000000000003908650337129266 \\
& 000000000003908650337111677
\end{aligned}
$$

This value is given by Flower, except that he makes the twenty-third his last figure 3 , not 1 . 
Again, in the case of a negative radix,

$$
\begin{aligned}
\log (1-.0009) & =\mu\left(-\frac{9}{104}-\frac{81}{2 \cdot 10^{8}}-\frac{729}{3 \cdot 10^{12}}-\frac{6561}{4 \cdot 10^{16}}-\right) \\
& =-.000900405243164 \times \mu \\
& =-.00039104102858 .
\end{aligned}
$$

To give an example of the use of series (ii).

$$
\begin{aligned}
& \text { Taking }(a+x)=3 \cdot 1416=264 \times 119 \times 10^{-4}, \\
& \quad(a-x)=3.14159265359 . \\
& 2 x=0.00000734641, \quad a=3.14159632679, \\
& 2 x / a=0.0000023384322 .
\end{aligned}
$$

$\log 3 \cdot 1416=1 \cdot 1447322242816$

23384322

Again, by series (iii),

$$
\text { Nat. } \log \pi=1 \cdot 1447 \overline{298858494}
$$

$$
\begin{gathered}
\log 8291=\log 8290+2 \mu\left(\frac{1}{16581}+\frac{1}{3}\left(\frac{1}{16581}\right)^{3}+\text { etc. }\right) . \\
\frac{3.918554530550273}{3.91860691514498} \\
\text { (To be continued.) }
\end{gathered}
$$

Sydney Lupton.

\section{MATHEMATICAL NOTES.}

404. [v. a. $\delta$ ]. A method of evaluating as a decimal any fraction of the form $\frac{1}{a \times 10^{b}+1}$, where $a$ is any integer, from 1 to 12 , and $b$ is any integer whatever.

This method is best explained by an illustration.

$$
\begin{gathered}
\frac{1}{401}+\frac{400}{401}=1=\dot{9} \text { and } \frac{1}{40 \mathrm{I}}=002 \ldots ; \\
\therefore \frac{400}{401}=997 \ldots .
\end{gathered}
$$

But $\quad \frac{1}{401}=\frac{400}{401} \div 400$

$$
\therefore \frac{1}{401}=00249 \ldots ; \quad \therefore \frac{400}{401}=99751 \ldots
$$

Continuing this process, we find

$$
\begin{aligned}
& \frac{1}{401}=\dot{0} 0249376558603491271820448877805486284289276807980049875311720 \\
& \text { 69825436408977556109725685785536159600997506234413965087281795 } \\
& 51122194513715710723192019950124688279301745635910224438902743 \\
& 1421446384039 \dot{9} \text {. } \\
& \frac{4}{4} 000=\dot{9} 9750623441396508728179551122194513715710723192019950124688279 \\
& 30174563591022443890274314214463840399002493765586034912718204
\end{aligned}
$$

405. [v. a. $\theta$; D. 6. b.] Inverse tabular functions.

The symbol $\sin ^{-1}, \cos ^{-1}$, etc., to denote "the angle whose sine, cosine, etc., is" generally proves a stumbling-block to learners, and to a small extent is inconsistent with the general interpretation of negative indices in algebra. On the continent the difficulty is evaded by writing arcsin, etc. I wish to propose as a substitute the prefix "anti," already used in this connection in 